\title{
Saturation of the magnetorotational instability by stable magnetoacoustic modes
}

\author{
Edward Liverts ${ }^{1}$, Yuri Shtemler ${ }^{1}$, Michael Mond ${ }^{1}$, \\ Orkan M. Umurhan ${ }^{2}$ and Dmitry V. Bisikalo ${ }^{3}$ \\ ${ }^{1}$ Department of Mechanical Engineering, Ben-Gurion University of the Negev, Beer-Sheva \\ 84105 , Israel \\ email: eliverts@bgu.ac.il, yshtemler@gmail .com,mond@bgu .ac.il \\ ${ }^{2}$ School of Natural Sciences, UC Merced, Merced, CA 95343, USA, and City College of San \\ Francisco, San Francisco, CA 94112, USA \\ email: oumurhan@ucmerced.edu \\ ${ }^{3}$ Institute of Astronomy of the Russian Academy of Science, 48 Pyatnitskaya, Moscow, Russia \\ email: bisikalo@inasan.rssi.ru
}

\begin{abstract}
The magnetorotational instability (MRI) of thin, vertically-isothermal Keplerian discs, under the influence of an axial magnetic field is investigated near the instability threshold. The nonlinear interaction of Alfven-Coriolis (MRI) modes with stable magnetoacoustic waves is considered. The transition of the Alfven-Coriolis modes to instability occurs when the linearized system has zero eigenvalue of multiplicity two. As a result the nonlinear ordinary differential equation that describes the evolution of the amplitude of the MRI mode near the threshold is of second order. Solutions of that amplitude equation reveal that the MRI is saturated to bursty periodical oscillations due to the transfer of energy to the stable magnetosonic modes.
\end{abstract}

Keywords. Accretion, magnetohydrodynamics, instabilities

\section{Introduction}

The destabilizing effect of an axial magnetic field on Couette flow has been discovered by Velikhov (1959) and Chandrasekhar (1960). Almost thirty years have passed before the importance of that phenomenon to astrophysics has been recognized, Balbus \& Hawley (1991), Balbus \& Hawley (2003). That mechanism, termed the magnetorotational instability (MRI). Knobloch \& Julien (2005) have described the nonlinear saturation of the MRI in a straight infinite vertically uniform channel with solid boundaries. In a complementary work, Umurhan et al. (2007) performed a weakly nonlinear analysis of the MRI close to marginality and showed that the MRI saturates due to dissipative effects to levels that scale with the square root of the magnetic Prandtl number.

\section{The physical model; linear and weakly nonlinear analysis}

The thin disk asymptotic expansion procedure is applied to the MHD equations in order to study the weakly nonlinear evolution of the MRI. The underlying physical property of the system is the supersonic nature of the Keplerian rotation whose Mach number is proportional to $1 / \epsilon$, where $\epsilon$ is the ratio of the disk's semi-thickness to its characteristic radius. Such procedure has been employed in numerous studies of thin disk dynamics, Regev (1983), Kluźniak \& Kita (1985), Umurhan et al. (2006), Shtemler et al. (2007), Shtemler et al. (2009) (see Shtemler et al. (2011) and Liverts et al. (2012a) for detailed derivation). The transition to instability (when plasma beta $\beta$ reaches $\beta_{c r}^{1}$ ) occurs when 
the linearized system of equations has a double zero eigenvalue. Thus, the appropriate amplitude equation is of the following form Liverts et al. (2012b):

$$
\frac{d^{2} a}{d t^{2}}=\gamma^{2} a\left(1-\frac{2}{5 \delta} a^{2}\right), \quad \gamma^{2}=\frac{27}{14} \delta
$$

where $\delta=\beta-\beta_{c r}^{1}$ is control parameter (supercriticality). The bifurcation diagram for this equation is depicted in Figure 1. Of particular importance is the fact that Eq.(2.1) is

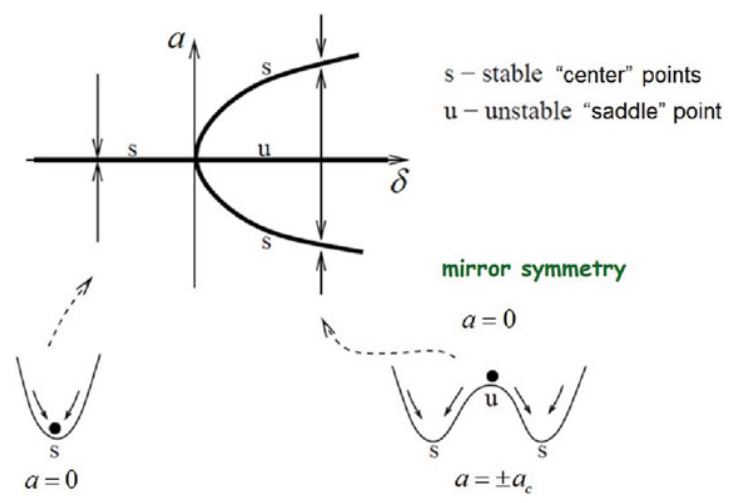

Figure 1. The bifurcation (supercritical) diagram.

derivable from an Hamiltonian and hence its solutions are bounded. This reflects indeed the saturation of the MRI even without any dissipation.

\section{Summary}

A novel non dissipative mechanism for the saturation of the MRI has been proposed. The latter imposes severe limitations on the efficiency of the MRI to directly generate significant levels of turbulence in thin accretion disks. This work was supported by grant number 180/10 of the Israel Science Foundation.

\section{References}

Velikhov, E. P. 1959, Soviet Physics JETP, 36, 995

Chandrasekhar, S. 1960, Proc. Natl. Acad. Sci., 46, 253

Balbus, S. A. \& Hawley, J. F. 1991, Astrophys. J., 376, 214

Balbus, S. A. \& Hawley, J. F. 2003, Annu. Rev. Astron. Astrophys., 41, 555

Knobloch, E. \& Julien, K. 2005, Phys. Fluids, 17, 094106

Umurhan, O. M., Menou, K., \& Regev, O. 2007, Phys. Rev. Lett., 98, 034501

Regev, O. 1983, A\&SA, 126, 146

Kluźniak, W. \& Kita, D. 1985, arXiv:astro-ph/0006266

Umurhan, O. M., Nemirovsky, A., Regev, O., \& Shaviv, G. 2006, A\& A, 446, 1

Shtemler, Y., Mond, M., \& Liverts, E. 2007, Astrophys. J., 665, 1371

Shtemler, Y., Mond, M., \& Rüdiger, G. 2009, MNRAS, 394, 1379

Shtemler, Y., Mond, M., \& Liverts, E. 2011, MNRAS, 413, 2957

Liverts, E. \& Mond, M. 2009, MNRAS, 392, 287

Liverts, E., Shtemler, Y., \& Mond, M. 2012a, AIP Proceedings no 1439, WISAP, P.L. Sulem, and M. Mond, Editors, 136

Liverts, E., Shtemler, Y., Mond, M., Umurhan, O. M., \& Bisikalo, D. V. 2012b, Phys. Rev. Lett., 109,224501 